\title{
Identifying predictors and evaluating the role of steroids in the prevention of post-embolization syndrome after transarterial chemoembolization and bland embolization
}

\author{
Rohit Agrawala, Muhammad Majeed ${ }^{a}$, Sheeba-Ba Aqeel ${ }^{a}$, Yuchen Wang ${ }^{b}$, Zohaib Haquea, \\ Yazan Abu Omara, Shristi Banskota Upadhyaya, Thomas Gastc, Bashar M. Attar ${ }^{b}$, Seema Gandhib \\ Cook County Health and Hospital System, IL, USA
}

Abstract

\begin{abstract}
Background Transarterial chemoembolization (TACE) and bland embolization (TAE), performed for hepatocellular carcinoma (HCC), are often complicated by post-embolization syndrome (PES). There are limited data regarding the incidence of PES after TAE and the role of steroids in PES. We report the incidence of PES post TACE and TAE, identify predictors, and evaluate the role of steroids in PES.
\end{abstract}

Methods Demographic and clinical variables of patients who underwent embolization were collected and PES was identified. Risk factors for PES, TACE and TAE were derived by logistic regression. We compared patients who received dexamethasone to those who did not, regarding baseline characteristics, occurrence of PES, and hospital stay.

Results A total of 171 patients, average age 60.5 years, underwent the procedure, $77.8 \%$ were male, and $87.7 \%$ had cirrhosis. Of these 171,107 underwent TACE and 64 TAE. Dexamethasone was given to 106 (61.9\%) patients, of whom 85 had TACE and 21 TAE. One hundred twenty-four patients $(72.5 \%)$ developed PES. PES occurred in more patients who underwent TACE, $80(74.7 \%)$ vs. $44(68.7 \%)$, and resulted in a longer hospital stay ( 1.47 vs. 1.12 days, $\mathrm{P}=0.034)$. Predictive factors for PES included female sex (odds ratio [OR] 2.76, 95\% confidence interval [CI] 1.04-7.34; $\mathrm{P}=0.041$ ), and alcohol-related HCC (OR 3.14, 95\%CI 1.42-6.95; $\mathrm{P}=0.005)$. Dexamethasone did not affect the length of hospital stay ( 1.43 vs. 1.29 days, $\mathrm{P}=0.422)$ or the rate of prolonged hospitalization ( $18.8 \%$ vs. $15.4 \%, \mathrm{P}=0.561)$.

Conclusion There was no difference in the incidence of PES following TACE or TAE and the use of dexamethasone did not reduce the incidence of PES or the duration of hospital stay.

Keywords Hepatocellular carcinoma, transarterial chemoembolization, bland embolization, post-embolization syndrome, dexamethasone

Ann Gastroenterol 2021; 35 (1): 1-6

a'Department of Medicine (Rohit Agrawal, Muhammad Majeed, Sheeba-Ba Aqeel, Zohaib Haque, Yazan Abu Omar, Shristi Banskota Upadhyay); ' Division of Gastroenterology and Hepatology, Department of Medicine (Bashar Attar, Seema Gandhi); 'Division of Radiology and Diagnostic Imaging (Thomas Gast), Cook County Health and Hospital System, IL, USA

\section{Conflict of Interest: None}

Correspondence to: Rohit Agrawal, Department of Medicine, Cook County Health and Hospital System, IL, USA,

e-mail: dr.rohitagrawal22@gmail.com

Received 24 May 2020; accepted 12 September 2020; published online 12 December 2020

DOI: https://doi.org/10.20524/aog.2020.0566

\section{Introduction}

Hepatocellular carcinoma (HCC) is the fifth most common cancer and has become the third most common cause of cancer-related mortality $[1,2]$. Despite the implementation of surveillance strategies in patients with known cirrhosis or hepatitis B, it is often diagnosed in its advanced stages, where curative treatments such as resection or liver transplantation are not a viable option. This has led to a significant interest in non-curative modalities as a suitable alternative [3].

While $85 \%$ of patients with HCC have incurable disease on presentation, there are several therapeutic options available, with arterial embolization considered to be the 
standard of care for patients with intermediate stage HCC [4]. Despite the chemoresistant nature of HCC, transarterial chemoembolization (TACE) is the most frequently performed arterial embolization and can also be used to bridge patients to liver transplantation $[2,5,6$,$] . Other commonly utilized options$ are transarterial embolization/bland embolization (TAE), drug-eluting bead TACE, and transarterial radioembolization. In a recent meta-analysis, which included 55 randomized controlled trials, arterial embolization was proved to have a survival advantage, but no benefit was noted of TACE over TAE [7].

While TACE is considered the standard of care for treating unresectable HCC, it does have the potential for complications such as post-embolization syndrome (PES). PES is a common complication of TACE and can be seen in 15-90\% of patients [8-11]. There is accumulating evidence suggesting that PES after TACE is associated with comparatively worse outcomes, including prolonged hospital stays and recurrent admissions [12,13]. Administration of steroids along with serotonin antagonists has been shown to reduce the incidence of PES after TACE; however, judicious use of these medications has other complications $[8,14]$. To the best of our knowledge, there are limited data about the incidence of PES after TAE and whether there is a potential role for steroids in the prevention of PES after bland embolization.

In this retrospective single-center study our aim was to: 1) report the incidence of PES in TACE and TAE; 2) identify the predictors of PES; and 3) evaluate the role of steroids in preventing PES in both TACE and TAE.

\section{Patients and methods}

\section{Database setup}

The electronic medical records (EMR) system at John H. Stroger Hospital in Cook County, Chicago, IL was searched for patients who underwent arterial embolization for HCC between January 2000 and January 2019. Identified patients were retrospectively followed for the occurrence of PES, defined as the occurrence of any one or more of the following within $48 \mathrm{~h}$ of procedure: A) new symptoms (fever, right upper quadrant pain, nausea, vomiting) which required pharmacotherapy; B) significant transaminitis (elevation of aspartate aminotransferase [AST] or alanine aminotransferase [ALT] to higher than twice the pre-procedure baseline value, and higher than upper normal limits); and C) new-onset decompensated liver disease (new-onset or re-accumulation of ascites requiring paracentesis; gastrointestinal bleeding proved on endoscopy to be secondary to esophageal variceal bleeding; and new-onset or worsened hepatic encephalopathy). Two physicians independently reviewed and confirmed the clinical endpoints to ensure the accuracy of the documentation. Patients with incomplete data as outlined below and patients without sufficient follow up (>30 days) post-procedure because of non-compliance were excluded from the analysis.
The present study was approved by the Institutional Review Board of the Cook County Health \& Hospitals System, Chicago. The database was set up and maintained by the Department of Gastroenterology and Hepatology, Cook County Health \& Hospitals System.

\section{Procedure and administration of dexamethasone}

The choice of TACE or TAE was decided by the interventional radiologist performing the procedure. As part of the preprocedural workup, every patient had a triple phase computed tomography or magnetic resonance imaging examination prior to the scheduled embolization. After informed consent and administration of sedation, a vascular sheath was introduced into the right common femoral artery using the Seldinger technique. The superior mesenteric and celiac arteries were catheterized, and angiograms and a portal venogram were then performed. Selective catheterization of the vessels supplying the tumor was performed using a microcatheter and wire. The vessels were then embolized with either polyvinyl alcohol particles or ethiodol oil, with or without the use of chemotherapeutic agents (doxorubicin vs. cisplatin). When embolization of the target vessel or vessels was complete, a final angiogram was performed. Patients were given steroids (dexamethasone $10 \mathrm{mg}$ every $8 \mathrm{~h}$ for one day, i.v.) at the radiologist's discretion.

\section{Variables}

We extracted variables including age, sex, presence of cirrhosis, etiologies of HCC (hepatitis B infection, hepatitis C infection, alcohol abuse), number and largest diameter of tumor at diagnosis, Barcelona staging of HCC, and prior treatment history. For further analysis, we categorized patients based on the type of intervention (TACE vs. TAE), prior procedure, and use of post-procedure dexamethasone.

\section{Statistical analysis}

We first described our cohort by summarizing the epidemiological variables, HCC-related information, and treatment-related information. The detailed description/ components of PES were summarized and compared between TACE and TAE groups. We then performed univariate and multivariate backward stepwise logistic regression to identify significant risk factors for the development of PES postprocedure. The same analysis was then repeated for TACE and TAE separately. We also compared patients who received dexamethasone to those who did not, regarding their baseline characteristics, occurrence of PES and hospital stay. All statistical analyses were performed using STATA (Version 14.0, College station, TX). We considered a P value $<0.05$ to be statistically significant. 


\section{Results}

\section{Cohort characteristics}

We identified a total of 171 patients who underwent arterial embolization for HCC during the study period and had complete laboratory and clinical data, as well as 30-day follow up (Table 1). The majority of the patients were male $(n=133,77.8 \%)$ and had cirrhosis $(n=150,87.7 \%)$ prior to the procedure. The average age was 60.5 years, with a standard deviation of 8.4 years.

The most prevalent etiology of HCC was alcohol abuse $(\mathrm{n}=89,52.1 \%)$, followed by hepatitis $\mathrm{C}$ infection $(\mathrm{n}=75,43.8 \%)$ and hepatitis B infection ( $n=40,23.4 \%)$, with overlaps among etiologies. Although half the patients $(n=84,50.3 \%)$ had only a solitary tumor at time of diagnosis, most $(n=135,79.5 \%)$ had relatively large tumors (largest diameter $>3 \mathrm{~cm}$ ), and $32.7 \%$ of patients had advanced cancer with Barcelona Stage C or D.

Of 171 patients who underwent arterial embolization, the majority underwent TACE $(n=107,62.5 \%)$ as opposed to TAE $(n=64,37.4 \%)$. After the procedure, dexamethasone (MOA dose) was given to 106 patients (61.9\%), 85 who underwent TACE and 21 who had TAE, using the timing and dosing described above.

\section{PES}

Of the 171 patients, 124 (72.5\%) developed PES, with a vast majority being diagnosed with PES as a result of consistent symptoms requiring pharmacotherapy. A significant portion of patients $(n=46,26.9 \%)$ had elevated AST or ALT levels compared to baseline, but only 3 patients were found to have decompensated liver disease (all cases developed ascites requiring paracentesis). The incidence of PES was slightly higher in patients who underwent TACE (80 of 107, 74.7\%) compared to TAE (44 of $64,68.7 \%$ ). However, the difference was statistically non-significant $(\mathrm{P}=0.394)$ (Table 2$)$.

We found that patients who developed PES had a longer hospital stay ( 1.47 vs. 1.12 days, $\mathrm{P}=0.034$ ) than those without PES. Similarly, the proportion of patients who required prolonged hospitalization (longer than $24 \mathrm{~h}$ inpatient stay) was also significantly higher in patients who developed PES $(20.9 \%$ vs. $8.5 \%, \mathrm{P}=0.050$ ).

\section{Prediction of PES}

In the univariate logistic regression, we identified alcoholrelated HCC as a potential risk factor for developing PES (odds ratio [OR] 2.15, 95\% confidence interval [CI] 1.08-4.29; $\mathrm{P}=0.028$ ). In contrast, cirrhosis (OR 0.11, 95\%CI 0.01-0.86; $\mathrm{P}=0.036$ ) and hepatitis $\mathrm{B}$ infection (OR 0.47, 95\%CI 0.21-0.98; $\mathrm{P}=0.045)$ appeared to be associated with a lower incidence of PES. In the backward stepwise multivariate logistic regression, independent predictive factors for PES included female sex (OR 2.76, 95\%CI 1.04-7.34; $\mathrm{P}=0.041$ ) and alcohol-related
Table 1 Baseline demographic and clinical data

\begin{tabular}{|c|c|}
\hline Cohort & $\mathrm{n}=171$ \\
\hline \multicolumn{2}{|l|}{ Epidemiology } \\
\hline Age (mean, SD) & $60.5(8.4)$ \\
\hline Sex $(\text { male })^{*}$ & $133(77.80 \%)$ \\
\hline \multicolumn{2}{|l|}{ Etiology* } \\
\hline Hepatitis $C^{*}$ & $75(43.80 \%)$ \\
\hline Hepatitis $B^{*}$ & $40(23.40 \%)$ \\
\hline Alcohol use $e^{\star}$ & $89(52.10 \%)$ \\
\hline Cirrhosis $^{*}$ & $150(87.70 \%)$ \\
\hline \multicolumn{2}{|l|}{ HCC } \\
\hline \multicolumn{2}{|l|}{ Number } \\
\hline Single ${ }^{*}$ & $84(50.30 \%)$ \\
\hline $2,3^{*}$ & $52(31.10 \%)$ \\
\hline$>3$ & $35(20.40 \%)$ \\
\hline \multicolumn{2}{|l|}{ Size } \\
\hline$<=3 \mathrm{~cm}^{*}$ & $35(20.50 \%)$ \\
\hline$>3 \mathrm{~cm}^{*}$ & $136(79.50 \%)$ \\
\hline \multicolumn{2}{|l|}{ Barcelona } \\
\hline $0, \mathrm{~A}, \mathrm{~B}$ & $115(67.25 \%)$ \\
\hline $\mathrm{C}, \mathrm{D}$ & $56(32.74 \%)$ \\
\hline \multicolumn{2}{|l|}{ Treatment } \\
\hline Systemic chemotherapy & $54(31.57 \%)$ \\
\hline \multicolumn{2}{|l|}{ Modality } \\
\hline TACE & $107(62.57 \%)$ \\
\hline TAE & $64(37.425)$ \\
\hline Multiple TACE & $84((49.12 \%)$ \\
\hline Dexamethasone & $106(61.98 \%)$ \\
\hline
\end{tabular}

*sample, percentage

$S D$, standard deviation; HCC, hepatocellular cancer; TACE, transarterial chemoembolization; TAE, bland embolization

Table 2 Incidence of PES in TACE vs. TAE

\begin{tabular}{lccc} 
Characteristics & Cohort & $\begin{array}{c}\text { TACE } \\
(\mathrm{N}=107)\end{array}$ & $\begin{array}{c}\text { TAE } \\
(\mathrm{N}=64)\end{array}$ \\
\hline Symptoms & 111 & 72 & 39 \\
$\quad$ Fever & 22 & 17 & 5 \\
$\quad$ Pain & 78 & 49 & 29 \\
Nausea/vomiting & 69 & 46 & 23 \\
Transaminitis & 46 & 31 & 15 \\
Complications \\
(ascites, HE, dialysis, die)
\end{tabular}

HCC (OR 3.14, 95\%CI 1.42-6.95; $\mathrm{P}=0.005)$. Moreover, the use of dexamethasone showed a trend of predicting PES (OR 2.1, 
95\% CI 0.99-4.47; $\mathrm{P}=0.053)$, rather than protecting against $\mathrm{PES}$ as anticipated (Table 3 ).

In subgroup multivariate analysis of patients undergoing TAE, female sex (OR 6.72, 95\%CI 1.3-34.72; $\mathrm{P}=0.023$ ) and alcohol-related HCC (OR 9.67, 95\%CI 2.22-42.13; $\mathrm{P}=0.003$ ) remained significant risk factors for PES, while dexamethasone administration did not show any significant effects (OR 1.53, 95\%CI 0.41-5.57; $\mathrm{P}=0.521$ ). Likewise, in patients with TACE, the use of dexamethasone did not show any benefit (OR 1.51, 95\%CI 0.54-4.23; $\mathrm{P}=0.427$ ).

\section{Effects of dexamethasone}

We analyzed the difference in baseline characteristics of patients treated with dexamethasone or not following arterial embolization, in an attempt to determine potential confounding factors that could falsely lead to the conclusion that dexamethasone is related to PES (Table 4). The 2 groups demonstrated statistically non-significant differences in the majority of epidemiological and HCC-related parameters, with the exception of a lower prevalence of alcohol-related HCC in patients who received dexamethasone $(45.3 \%$ vs. $63.1 \%$, $\mathrm{P}=0.024)$. There were no differences in the majority of baseline laboratory values, except for a slightly higher serum total bilirubin ( 1.16 vs. $0.94 \mathrm{mg} / \mathrm{dL}, \mathrm{P}=0.046$ ).

There were also no differences in the length of average hospital stay ( 1.43 vs. 1.29 days, $\mathrm{P}=0.422$ ) or the percentage of prolonged hospitalizations ( $18.8 \%$ vs. $15.4 \%, \mathrm{P}=0.561)$, which casts further doubt on the utility of dexamethasone in this clinical setting.

\section{Discussion}

PES, an inflammatory syndrome commonly seen after embolization, is thought to be mediated by inflammatory cytokines released as a result of embolization-induced liver necrosis and/or chemotherapeutic agents $[8,15]$. According to our analysis, $75 \%$ of the patients in our cohort who underwent TACE developed PES, comparable to the rates reported in the literature. Similarly, $70 \%$ of the patients who underwent TAE developed PES; to the best of our knowledge, this is the first study to report the incidence of PES after TAE. The fact that a similar number of patients were affected, even with TAE, suggests that the mechanism of PES is probably driven by liver necrosis due to interruption of blood flow, rather than by chemotherapeutic agents. PES is considered an inevitable consequence of embolization [16] and there are data suggesting it may be a predictor of overall survival. It is unclear whether the consequences of PES are due to the underlying HCC burden or to the occurrence of PES per se. Mason et al reported a 2-fold greater risk of death in patients undergoing TACE who developed PES [12], and other studies reported greater mortality in patients who developed PES $[17,18]$. In our analysis, no such association was found, as none of our patients died within 30-day follow up. However, PES can lead

Table 3 Univariate and multivariate regression to identify predictors of PES

\begin{tabular}{|c|c|c|c|c|}
\hline \multirow[t]{2}{*}{ Cohort } & \multicolumn{2}{|c|}{ Univariate } & \multicolumn{2}{|l|}{ Multivariate } \\
\hline & $\begin{array}{c}\text { Odds ratio } \\
(95 \% \text { confidence interval) }\end{array}$ & P-value & $\begin{array}{c}\text { Odds ratio } \\
(95 \% \text { confidence interval) }\end{array}$ & $\mathrm{P}$-value \\
\hline Advanced age & $1.06(0.49-2.27)$ & 0.886 & & \\
\hline Sex & $1.55(0.65-3.69)$ & 0.316 & $2.76(1.04-7.34)$ & 0.041 \\
\hline Cirrhosis & $0.11(0.01-0.86)$ & 0.036 & $0.12(0.01-0.96)$ & 0.046 \\
\hline Advanced Barcelona stage (C/D) & $0.81(0.39-1.63)$ & 0.558 & & \\
\hline Hepatitis B & $0.47(0.21-0.98)$ & 0.045 & & \\
\hline Hepatitis C & $1.55(0.77-3.09)$ & 0.214 & & \\
\hline Alcohol use & $2.15(1.08-4.29)$ & 0.028 & $3.14(1.42-6.95)$ & 0.005 \\
\hline TACE vs. TAE & $0.74(0.37-1.47)$ & 0.396 & & \\
\hline Multiple procedure & $1.44(0.73-2.83)$ & 0.291 & & \\
\hline Dexamethasone & $1.47(0.74-2.90)$ & 0.27 & $2.1(0.99-4.47)$ & 0.053 \\
\hline \multicolumn{5}{|l|}{ TAE } \\
\hline Sex & $6.72(1.3-34.72)$ & 0.023 & & \\
\hline Alcohol use & $9.67(2.22-42.13)$ & 0.003 & & \\
\hline Dexamethasone & $1.53(0.41-5.57)$ & 0.521 & & \\
\hline \multicolumn{5}{|l|}{ TACE } \\
\hline Dexamethasone & $1.51(0.54-4.23)$ & 0.427 & & \\
\hline
\end{tabular}

PES, post-embolization syndrome; TACE, transarterial chemoembolization; TAE, bland embolization 
Table 4 Differences in demographics, clinical data and length of hospital stay in relation to dexamethasone use

\begin{tabular}{lccc}
\hline Characteristics & Non-Dexamethasone & Dexamethasone & P-value \\
\hline Age & 59.6 & 61.1 & 0.246 \\
Male sex & $76.9 \%$ & $78.3 \%$ & 0.833 \\
Etiology & & & 0.767 \\
Hepatitis B & $24.6 \%$ & $22.6 \%$ & 0.152 \\
Hepatitis C & $36.9 \%$ & $48.1 \%$ & 0.024 \\
Alcohol use & $63.1 \%$ & $45.3 \%$ & 0.333 \\
Cirrhosis & $84.6 \%$ & $89.6 \%$ & 0.014 \\
Advanced Barcelona & $78.5 \%$ & $60.4 \%$ & 0.046 \\
Pre-procedure laboratory values & & & 0.917 \\
Total bilirubin & 0.94 & 1.16 & 0.482 \\
AST & 85.8 & 87.2 & 0.534 \\
ALT & 73.7 & 66.7 & 3.79 \\
Albumin & 3.64 & 14.3 & 0.464 \\
PT & 14.6 & 1.36 & 0.499 \\
Creatinine & 1.26 & 1.43 & 0.422 \\
Hospitalization & 1.29 & $18.8 \%$ & 0.561 \\
Prolonged hospitalization & $15.4 \%$ & & \\
\hline
\end{tabular}

AST, aspartate aminotransferase; $A L T$, alanine aminotransferase; $P T$, prothrombin time

to a prolonged hospital stay, as around $20 \%$ of the patients who developed PES required hospitalization for more than $24 \mathrm{~h}$, compared to $8 \%$ of the patients without PES [8]. Our results are comparable to those of a study by Leung et al, which reported that $40 \%$ of patients who developed PES had prolonged hospital stays [13]. Prolonged hospitalization can lead to a significant financial burden. Moreover, the discomfort from TACE can discourage subsequent TACE and can result in over-utilization of hospital resources for the evaluation of alternative etiologies that can mimic the symptoms of PES [8].

As the development of PES can have unfavorable outcomes, it is important to identity the predictors of PES to offer alternate therapies or prophylactic medications to prevent PES in highrisk populations. We identified female sex and alcohol-related HCC as potential risk factors for developing PES in patients undergoing TACE. However, no such risk factors were identified for TAE. A previous study that looked at 70 TACE procedures in 29 patients found that gallbladder embolization increased the risk of PES, while repeat TACE in a previously embolized vessel decreased the risk of PES [13]. Similarly, Khalaf et al identified previous PES, large tumor burden, and drug-eluting embolic TACE as significant predictors for the development of PES [19]. PES following TAE has not been studied exclusively, but in a study published by Patel et al, which included 62 patients of whom $66.1 \%$ underwent TAE alone, the predictor of post-embolization pain and nausea was pre-procedure liver function tests [20]. Identification of predictors can aid in the risk-stratification of patients, and those at high risk could be selected for TAE to avoid greater medical costs and burdens.

We also investigated whether dexamethasone administration may reduce the incidence of PES. There are convincing reports in the literature that the use of dexamethasone can prevent PES. In a recent randomized controlled trial of 120 patients undergoing TACE, Ogasawara et al demonstrated that dexamethasone was over 4 times more effective than placebo in preventing PES [21]. Similarly, a study by Yinglu et al also favored the use of steroids, particularly in combination with ginsenosides, to prevent and treat PES after TACE. A retrospective review published in 2012 did not show a benefit of dexamethasone in reducing analgesic use, but it reduced the post-TACE use of antiemetics [22]. However, our analysis did not show any beneficial effect of dexamethasone in preventing PES in TACE, which could be related to the lower dose of dexamethasone used in our practice. Ogasawara et al administered dexamethasone for 3 days postprocedure; however, in our practice patients received it for only one day [21]. Moreover, we did not find any benefit with prophylactic dexamethasone in patients undergoing TAE, and to the best of our knowledge this is the first study to document such a potential lack of efficacy.

This study had several limitations. It included a small sample of 171 patients from a single center, which reduced the power to detect a significant difference if present. Likewise, because of its retrospective nature, the study was subject to certain biases. We used clinical data to identify patients with PES, which could have over- or underestimated the rate of PES. We also acknowledge the selection bias imposed by dexamethasone use at the interventional radiologist's discretion, even though these patients' demographic and clinical variables were fairly similar, as shown in Table 4. This, however, does not account for all variables that may affect the outcome.

In conclusion, we found no difference in the incidence of PES following TACE or TAE, and the routine use of 
dexamethasone did not appear to reduce the incidence of PES or the duration of hospital stay. We identified the presence of alcohol-related HCC, cirrhosis, hepatitis B and female sex as potential predictors for development of PES.

\section{Summary Box}

\section{What is already known:}

- Transarterial chemoembolization (TACE) and bland embolization (TAE) are frequently performed to treat hepatocellular carcinoma (HCC), often complicated by post-embolization syndrome (PES)

- PES is considered an inevitable consequence of embolization, occurring in $15-90 \%$ of the patients and there are data suggesting it may be a predictor of overall survival

- Administration of steroids along with serotonin antagonists has been shown to reduce the incidence of PES after TACE; however, the judicious use of these medications has other complications

\section{What the new findings are:}

- $75 \%$ and $70 \%$ of the patients in our cohort who underwent TACE and TAE, respectively, developed PES

- Female sex and alcohol-related HCC were potential risk factors for developing PES in patients undergoing TACE; however, no such risk factors were identified for TAE

- There were no differences in the length of average hospital stay or the percentage of prolonged hospitalizations with the use of dexamethasone

\section{References}

1. Petrick JL, Braunlin M, Laversanne M, Valery PC, Bray F, McGlynn KA. International trends in liver cancer incidence, overall and by histologic subtype, 1978-2007. Int J Cancer 2016;139:1534-1545.

2. Marrero JA, Kulik LM, Sirlin CB, et al. Diagnosis, staging, and management of hepatocellular carcinoma: 2018 practice guidance by the American Association for the Study of Liver Diseases. Hepatology 2018;68:723-750.

3. Davila JA, Henderson L, Kramer JR, et al. Utilization of surveillance for hepatocellular carcinoma among hepatitis C virus-infected veterans in the United States. Ann Intern Med 2011;154:85-93.

4. Roxburgh P, Evans TR. Systemic therapy of hepatocellular carcinoma: are we making progress? Adv Ther 2008;25:1089-1104.

5. Simonetti RG, Liberati A, Angiolini C, Pagliaro L. Treatment of hepatocellular carcinoma: a systematic review of randomized controlled trials. Ann Oncol 1997;8:117-136.

6. Davila JA, Duan Z, McGlynn KA, El-Serag HB. Utilization and outcomes of palliative therapy for hepatocellular carcinoma: a population-based study in the United States. J Clin Gastroenterol 2012;46:71-77.

7. Katsanos K, Kitrou P, Spiliopoulos S, Maroulis I, Petsas T, Karnabatidis D. Comparative effectiveness of different transarterial embolization therapies alone or in combination with local ablative or adjuvant systemic treatments for unresectable hepatocellular carcinoma: a network meta-analysis of randomized controlled trials. PLoS One 2017;12:e0184597.

8. Piscaglia F, Tovoli F, Pini P, Salvatore V. A new horizon in the prevention of the postembolization syndrome after transcatheter arterial chemoembolization for hepatocellular carcinoma. Hepatology 2018;67:467-469.

9. Chung JW, Park JH, Han JK, et al. Hepatic tumors: predisposing factors for complications of transcatheter oily chemoembolization. Radiology 1996;198:33-40.

10. Dhand S, Gupta R. Hepatic transcatheter arterial chemoembolization complicated by postembolization syndrome. Semin Intervent Radiol 2011;28:207-211.

11. Wigmore SJ, Redhead DN, Thomson BN, et al. Postchemoembolisation syndrome--tumour necrosis or hepatocyte injury? Br J Cancer 2003;89:1423-1427.

12. Mason MC, Massarweh NN, Salami A, Sultenfuss MA, Anaya DA. Post-embolization syndrome as an early predictor of overall survival after transarterial chemoembolization for hepatocellular carcinoma. HPB (Oxford) 2015;17:1137-1144.

13. Leung DA, Goin JE, Sickles C, Raskay BJ, Soulen MC. Determinants of postembolization syndrome after hepatic chemoembolization. $J$ Vasc Interv Radiol 2001;12:321-326.

14. Ishikawa T. Prevention of post-embolization syndrome after transarterial chemoembolization for hepatocellular carcinomais prophylactic dexamethasone useful, or not? Hepatobiliary Surg Nutr 2018;7:214-216.

15. Clark TW. Complications of hepatic chemoembolization. Semin Intervent Radiol 2006;23:119-125.

16. Basile A, Carrafiello G, Ierardi AM, Tsetis D, Brountzos E. Quality-improvement guidelines for hepatic transarterial chemoembolization. Cardiovasc Intervent Radiol 2012;35:765-774.

17. Pinato DJ, Sharma R. An inflammation-based prognostic index predicts survival advantage after transarterial chemoembolization in hepatocellular carcinoma. Transl Res 2012;160:146-152.

18. McNally ME, Martinez A, Khabiri $\mathrm{H}$, et al. Inflammatory markers are associated with outcome in patients with unresectable hepatocellular carcinoma undergoing transarterial chemoembolization. Ann Surg Oncol 2013;20:923-928.

19. Khalaf MH, Sundaram V, AbdelRazek Mohammed MA, et al. A predictive model for postembolization syndrome after transarterial hepatic chemoembolization of hepatocellular carcinoma. Radiology 2019;290:254-261.

20. Patel NH, Hahn D, Rapp S, Bergan K, Coldwell DM. Hepatic artery embolization: factors predisposing to postembolization pain and nausea. J Vasc Interv Radiol 2000;1 1:453-460.

21. Ogasawara S, Chiba T, Ooka Y, et al. A randomized placebocontrolled trial of prophylactic dexamethasone for transcatheter arterial chemoembolization. Hepatology 2018;67:575-585.

22. Kogut MJ, Chewning RH, Harris WP, Hippe DS, Padia SA. Postembolization syndrome after hepatic transarterial chemoembolization: effect of prophylactic steroids on postprocedure medication requirements. J Vasc Interv Radiol 2013;24:326-331. 